\title{
Nanoparticulate Carriers of Anticancer Drugs and Therapeutics
}

\author{
Ignác Capek* \\ Institute of Measurement Sciences SAS, Bratislava, Slovakia
}

*Corresponding author: Ignác Capek, Institute of Measurement Sciences SAS, Bratislava, Slovakia.

Received Date: March 28, 2019

Published Date: April 08, 2019

\section{Mini Review}

Nanoscale drug delivery platforms have been developed over the past four decades that have shown promising clinical results in several types of cancer and inflammatory disorders. Nanoparticles have special characteristics of high surface to volume ratio, size controllability, and can be easily functionalized during synthesis [1]. Biodegradable polymer nanoparticles have been used as carriers for drugs, peptides, proteins, vaccines, and nucleotides [2]. Nanoparticles (NPs) can protect drug moieties from degradation and provide sustained drug release. NPs are sometimes effective in facilitating intracellular delivery of bioactive materials. Polylactideco-glycolic acid (PLGA) NPs have been used for various applications in vaccination, cancer therapy, and the treatment of cerebral disorders [3].

In particular, NPs have been widely investigated for use in cancer therapy. NPs, if given intravenously, can extravasate into and accumulate within tumor tissues that have defective blood vessels and impaired lymphatic drainage [4]. This enhanced permeability and retention (EPR) effect helps direct nanoparticles to tumor sites [5]. Even though a lot of polymer nanoparticles have emerged as a promising carrier, they have a number of intrinsic drawbacks. These nanocarriers carrying therapeutic payloads are maximizing the therapeutic outcomes while minimizing adverse effects. The functionality of bare (polymer, metal, quantum dots, silica, etc.) nanoparticles is limited to drug depot or drug solubilization in their hard cores. When these systems are administered intravenously, the nanoparticles undergo rapid clearance from systemic circulation before reaching the site of action. Recent research shows that surface functionalization of nanoparticles and development of new nanoparticulate dosage forms help overcome these delivery challenges and improve in vivo performance.

The objectives of these strategies are to improve their functionality and in vivo performance. Functionalization of polymer nanoparticles includes surface modification by PEGylation (PEG, polyethylene glycol), lipid- or surfactant-coating, polyion complexation, and conjugation with cell-targeting ligands. Functional polymer-based nanoparticulate systems offer various beneficial outcomes, such as: minimization of opsonin adsorption, prolongation of blood residence time, reduction of drug side effects, elimination of the use of toxic adjuvants for drug solubilization, improved targeted drug delivery, efficient cellular translocation and subcellular trafficking, and synergic drug combination therapy [6]. PEGylation technology contributes to (a) increasing the aqueous solubility and stability, (b) reducing intermolecular aggregation, (c) decreasing immunogenicity, and (d) prolonging the systemic circulation time of a compound. PEG conjugation to various hydrophobic groups or polymers enables the preparation of self-assembling micelles. In addition, the end groups of PEG can be modified to aldehyde, amino, carboxyl, or methyl groups to alter the surface charge of a nanoparticulate carrier [7].

These PEG derivatives are used for conjugation with ligands that have amine, thiol, or carboxyl groups. Due to their amphiphilic nature, when block copolymers are dispersed in an aqueous medium, they self-assemble into micellar forms. PEG acts as a hydrophilic corona, while the second block serves as a hydrophobic core. The hydrophobicity of polymer cores can be increased by chemical derivatization. For example, mono-hexyl and di-hexyl substituted lactide monomers have been used to produce hexylPLGA via ring-opening polymerization [8]. This technology makes it possible to prepare 20-80 nm micelles. PEG-hexyl-PLGA micelles, due to their elevated hydrophobicity, have greater capacity for loading hydrophobic drugs than do typical PEG-PLGA micelles. For example, the aqueous solubility of cyclosporine A is $0.01 \mathrm{mM}$, but its solubility in PEG-hexyl-PLGA micelles increases to $1.10 \mathrm{mM}$ [9]. Methoxy poly (ethylene glycol)-poly (lactic acid) copolymer (mPEG-PLA) micelles encapsulated hydrophobic curcumin via a 
disulfide bond [10]. The extent of curcumin release from disulfide bond-linked micelles was nearly three times higher compared to the control micelles. Such rapid release led to a lower half maximal inhibitory concentration (IC50) in HeLa cells at $~ 18.5 \mu \mathrm{g} \mathrm{mL}-1$, whereas the IC50 of control micelles was $\sim 41.0 \mu \mathrm{g} \mathrm{mL}-1$.

The cellular uptake study also revealed higher fluorescence intensity for redox-sensitive micelles. The redox-sensitive polymeric conjugate micelles could enhance curcumin delivery while avoiding premature release and achieving on-demand release under the high glutathione concentration in the cell cytoplasm. Curcumin, diferuloylmethane polyphenol, has been associated with multiple health benefits, including cancer prevention by its ability to modulate intracellular signaling pathways implicated in inflammation, proliferation, invasion, survival, and apoptosis. It has various therapeutic properties such as anti-inflammatory, antimicrobial, antitumor, antiparasitic and antioxidant properties [11]. Curcumin possesses anti-inflammatory effects that retard hepatic fibrosis and cirrhosis in carbon tetrachloride (CCl4)-caused liver fibrosis [12]. This herbal antioxidant alleviates fibrosis through the effects of reduced activation, retarded mobility, and angiogenetic activity on hepatic stellate cells (HSCs) [13]. Curcumin suppresses HSC activation through disruption of transforming growth factor- $\beta$ (TGF- $\beta$ ) signaling [14] and activation of peroxisome proliferatoractivated receptor-c (PPAR-c) [15]. Approaches that increase curcumin bioavailability have been suggested, and nanomedicine appears to be a feasible way to increase the efficacy of curcumin [16].

A further example includes PEGylated micelles/nanoparticles (polyethylene glycol (PEG)), polyplexes, polymersomes, polylactideco-glycolic acid (PLGA)- based nanocomposites (core-shell-type lipid-PLGA hybrids, cell-PLGA hybrids, receptor-specific ligandPLGA conjugates) and nanoparticulate systems, therapeutics and theranostics. Each PLGA-based nanoparticulate dosage form has specific features that distinguish it from other nanoparticulate systems. The characteristics of PLGA-based nanostructures are also controlled by the stereochemistry of lactic acid (D, L, or DL), degree of crystallinity, lactic acid/glycolic acid ratio, and molecular weight. PLGA is available with different end groups, namely a free carboxylic end group and an ester-terminated group [17]. The PLGApolyethyleneimine (PEI) micelles showed better translocation into human keratinocytes than plain PLGA nanoparticles. It is likely that such improvement in cellular uptake arose from the cationic surface of PEI-PLGA micelles and the strong affinity of PEI to lipopolysaccharides and cell membranes, as has been suggested elsewhere [18].

A poly(L-cysteine)-b-PLGA copolymer, linked through a disulfide bond, was formulated into a micellar dosage form [19]. The hydrophilic polyamino acid domain formed a corona around the hydrophobic PLGA core. The chain lengths of block polymers and the redox status of the disulfide bond affected the size of the polymeric micelles. Other polypeptides made of aspartic acid, glutamic acid, and lysine have also been used as coronary segments of PLGA-based micelles [20]. For example, carboxylic end groups of PLGA chains were reacted with primary $\varepsilon$-amino groups of a cationic poly(L-lysine) (PLL), to produce PLGA-g-PLL copolymers of various grafting percentages $(3.6 \%-8.0 \%)$ [21]. These conjugates self-assembled into $70-80 \mathrm{~nm}$ micelles in water. These polymeric micelles are more thermodynamically stable and have lower critical micelle concentrations than typical surfactant micelles. Discussed functional PEG, PLGA, PLL, etc. -based nanoparticulate systems are expected to deliver chemotherapeutic, diagnostic, and imaging agents in a highly selective and effective manner.

\section{Acknowledgment}

This research is supported by the VEGA project No. 2/0164/17.

\section{Conflict of Interest}

No conflict of interest.

\section{References}

1. Vakurov A, Pchelintsev NA, Forde J, Fagain CO, Gibson T, et al. (2009) The preparation of size-controlled functionalized polymeric nanoparticles in micelles. Nanotechnology 20: 295605-295612.

2. Panyama J, Labhasetwar V (2003) Biodegradable nanoparticles for drug and gene delivery to cells and tissue. Adv Drug Deliv Rev 55(3): 329-347.

3. Danhier F, Ansorena E, Silva JM, Coco R, Breton AL, et al. (2012) PLGAbased nanoparticles: An overview of biomedical applications. J Control Release 161(2): 505-522.

4. Hobbs SK, Monsky WL, Yuan F, et al. (1998) Regulation of transport pathways in tumor vessels: role of tumor type and microenvironment. Proc Natl Acad Sci USA, 95(8): 4607-4612.

5. Maeda H, Wu J, Sawa T, Matsumura Y, Hori K (200) Tumor vascular permeability and the EPR effect in macromolecular therapeutics: a review. J Control Release 65(1-2): 271-284.

6. Peer D, Karp JM, Hong S, Farokhzard OC, Margalit R, et al. (2007) Nanocarriers as an emerging platform for cancer therapy. Nat Nanotechnol 2(12): 751-760.

7. Wonganan P, Croyle MA (2010) PEGylated adenoviruses: from mice to monkeys. Viruses, 2(2): 468-502.

8. Trimaille T, Gurny R, Möller M (2007) Poly (hexyl-substituted lactides): novel injectable hydrophobic drug delivery systems. J Biomed Mater Res A 80(1): 55-65.

9. Kasimova AO, Pavan GM, Danani A, et al. (2012) Validation of a novel molecular dynamics simulation approach for lipophilic drug incorporation into polymer micelles. J Phys Chem B 116(14): 43384345.

10. Cao Y, Gao M, Chen C, Fan A, et al. (2015) Triggered-release polymeric conjugate micelles for on-demand intracellular drug delivery. Nanotechnology 26: 115101-115110.

11. Salem M, Rohani S, Gillies ER (2014) Curcumin, a promising anticancer therapeutic: a review of its chemical properties, bioactivity and approaches to cancer cell delivery. RSC Advances 4: 10815-10829.

12. Fu Y, Zheng S, Lin J, Ryerse J, Chen A (2008) Curcumin protects the rat liver from CCl4-caused injury and fibrogenesis by attenuating oxidative stress and suppressing inflammation. Mol Pharmacol 73: 399-409.

13. Zhang F, Zhang Z, Chen L, Kong D, Zhang X, et al. (2015) Curcumin attenuates angiogenesis in liver fibrosis and inhibits angiogenic properties of hepatic stellate cells. J Cell Mol Med 18: 1392-1406.

14. Zheng S, Chen A (2007) Disruption of transforming growth factorbeta signaling by curcumin induces gene expression of peroxisome proliferator-activated receptor-gamma in rat hepatic stellate cells. Am J Physiol Gastrointest Liver Physiol 292: G113-G123. 
15. Lin J, Chen A (2008) Activation of peroxisome proliferator-activated receptor-gamma by curcumin blocks the signaling pathways for PDGF and EGF in hepatic stellate cells. Lab Investig J Tech Methods Pathol 88: 529-540.

16. Yallapu MM, Jaggi M, Chauhan SC (2012) Curcumin nanoformulations: A future nanomedicine for cancer. Drug Discov Today 17: 71-80.

17. Houchin ML, Topp EM (2008) Chemical degradation of peptides and proteins in PLGA: A review of reactions and mechanisms. J Pharm Sci 97(7): 2395-2404.

18. Kirchelis R, Wightman L, Wagner E (2001) Design and gene delivery activity of modified polyethyleneimines. Adv Drug Deliv Rev 53(3): 341358.
19. Sun J, Chen X, Lu T, et al. (2008) Formation of reversible shell crosslinked micelles from the biodegradable amphiphilic diblock copolymer poly(L-cysteine)-block-poly(L-lactide). Langmuir 24(18): 10099-10106.

20.Sun J, Deng C, Chen X, et al. (2007) Self-assembly of polypeptidecontaining ABC-type triblock copolymers in aqueous solution and its $\mathrm{pH}$ dependence. Biomacromolecules 8(3): 1013-1017.

21. Jeong JH, Byun Y, Park TG. (2003) Synthesis and characterization of poly(L-lysine)-g-poly (D, L-lactic-co-glycolic acid) biodegradable micelles. J Biomater Sci Polym Ed 14(1): 1-11. 